\title{
Propagation of nitrogen gas in a liquid helium cooled vacuum tube following sudden vacuum loss- Part I: Experimental investigations and analytical modeling
}

\author{
R. C. Dhuley ${ }^{1,2}$ and S. W. Van Sciver ${ }^{1,2}$ \\ ${ }^{1}$ National High Magnetic Field Laboratory, Tallahassee, FL 32310 \\ ${ }^{2}$ Mechanical Engineering Department, FAMU-FSU College of Engineering, Tallahassee, FL 32310
}

\begin{abstract}
This paper describes propagation of near atmospheric nitrogen gas that rushes into a liquid helium (LHe) cooled vacuum tube after the tube suddenly loses vacuum. The loss-of-vacuum scenario resembles accidental venting of atmospheric air to the beam-line of a superconducting particle accelerator and is investigated to understand how the in-flowing air will propagate in such geometry. In controlled experiments, we simulated loss of vacuum by rapidly venting a large reservoir of nitrogen gas (a substitute for air) to a vacuum tube immersed in a LHe bath at $4.2 \mathrm{~K}$. The resulting rise in the tube pressure and temperature were measured by pressure probes and thermometers arranged along the tube length. The data show that the propagation of nitrogen gas in the LHe cooled vacuum tube is orders of magnitude slower than in the same tube at room temperature. Interestingly, the gas front speed in the LHe cooled tube also decreases along the tube. A gas propagation model developed by employing conservation of mass identifies mass transfer (gas condensation in the tube) as the principal cause of the slow propagation as well as of the front deceleration. Some limitations of this analytical model are discussed in the context of quantifying the propagation speed. The speed obtained from direct measurements is seen to decrease exponentially along the tube. This exponential decay form of the propagation speed well represents the data from experiments that have different mass flow rates of nitrogen gas flowing into the vacuum tube after venting.
\end{abstract}

Keywords: Accidental vacuum loss, propagation speed, superconducting, particle accelerator, beam-line

\section{Introduction}

When a long channel holding vacuum at room temperature is abruptly opened to atmosphere, the inflowing air will cause a pressure front to propagate down the channel. The front speed in this scenario can be measured by means of an array of pressure probes placed in the channel [1]. The theoretical maximum speed of a gas propagating in a perfect vacuum, called as 'escape speed', is given by $2 a /(\gamma-1)$ [2]. In this expression, $a$ is the speed of sound in the gas at its source and $\gamma$ is the ratio of specific heats. For air at room temperature, the escape speed is nearly $1700 \mathrm{~m} / \mathrm{s}$. This theory, however, assumes perfect vacuum in the channel and that the density of the gas front adjacent to the vacuum is zero. Due to this vanishing density, no pressure measurement apparatus with a finite measurement resolution can record the theoretical speed of the front. Nonetheless, in realistic experiments such as by Takiya [3] the front speed in a vacuum channel (pressure $\approx 10^{-3} \mathrm{~Pa}$ ) was recorded to be as high as $700 \mathrm{~m} / \mathrm{s}$. Although substantially lower than the theoretical maximum, this speed is still nearly twice the speed of sound in air at room temperature. 
The beam-line of a superconducting particle accelerator (European Spallation Source for example) is a special case of a high vacuum channel (pressure $\approx 10^{-8} \mathrm{~Pa}$ ), which is cooled by immersion in liquid helium (LHe) [4]. When such a channel suddenly loses its vacuum to atmospheric air, the air front is observed to propagate at speeds substantially lower than in vacuum channels at room temperature. Past research [5,6] that investigated vacuum loss in LHe cooled channels has reported this speed to be of the order of $10 \mathrm{~m} / \mathrm{s}$. This order of magnitude reduction in the propagation speed is believed to be due to the intensive mass and heat transfer from the in-flowing warm air $(\approx 295 \mathrm{~K})$ to the extremely cold walls of the channel $(4.2 \mathrm{~K}$ or colder). In this paper we present experimental and analytical findings that elucidate the role of this heat and mass transfer in slowing the propagation of nitrogen gas (a substitute of air) in a LHe cooled channel. An experimental apparatus [7] has been devised to simulate a sudden loss of vacuum in a $4.2 \mathrm{~K} \mathrm{LHe}$ cooled tube and to measure the resulting rise in temperature and pressure along the tube as the gas propagates. The influence of mass and heat transfer on the propagation is investigated experimentally. We explain some characteristics of gas propagation with an analytical model formulated using conservation of mass. Some limitations in evaluating the propagation speed using the model are described and then the propagation speed is obtained directly from the measured data. Our analysis and experiments provide first insights into the nature of the loss-of-vacuum induced air propagation in a LHe cooled vacuum channel.

\section{Experimental Setup and Procedure}

Figure 1 shows the general characteristics of the experimental apparatus. The propagation measurement site is a vacuum tube (OFHC copper, length $1.5 \mathrm{~m}$, inner diameter $32 \mathrm{~mm}$, and wall thickness $3 \mathrm{~mm}$ ) immersed in a bath of LHe at its normal boiling temperature of $4.2 \mathrm{~K}$. Accidental loss of vacuum is simulated by opening the solenoid valve (SV) ( 25 ms opening time), which vents a tank (85 liters in volume) containing nitrogen gas to the cold vacuum tube. The tube is equipped with an array of miniature pressure probes on the inside and thermometers embedded in the tube wall from the outside. These sensors record the arrival times of the gas front at sequential locations along the tube. The gas propagation speed is deduced from the separation between the sensors and the measured arrival times. The gas flowing from the supply tank to the vacuum tube is made to choke using a venturi tube so as to generate a near constant mass flow in to the vacuum tube. Note that although the venturi chokes after opening the SV, the mass in-flow rate gradually decreases as the tank depressurizes with time. However, this decrease is small so that simple time averaging provides a reasonable estimate for the near-constant mass in-flow rate. The mass in-flow rate is determined from the tank de-pressurization rate recorded by a pressure transducer placed inside the gas tank. The mass in-flow rate calculation and averaging are elaborated in the Appendix. A variety of mass flow rates can be obtained from this configuration by starting with different pressures of the gas in the supply tank.

The installation techniques for the pressure probes and the thermometers to ensure their proper operation in the cryogenic environment have been elaborated in [7]. The pressure sensors are Kulite XCQ-092 probes with 0-100/150 kPa range of operation. All pressure probes were calibrated using MKS 626Baratron capacitance manometer as a reference. During experiments, the pressure probes were excited at 10 VDC using TENMA 72-7245 voltage supply and the probe output voltages were measured on National Instruments USB-6225 DAQ at a sampling rate of $20 \mathrm{kHz}$ per probe. The uncertainty in pressure measurement is $\pm 20 \mathrm{~Pa}$ in the entire operating range and is dominated by electromagnetic interference (noise). Lake Shore Cernox ${ }^{\circledR} 1050$ SD thermometers measured the tube temperature. These RTDs were calibrated in the temperature range of 2-80 K referenced to a factory calibrated thermometer. During 


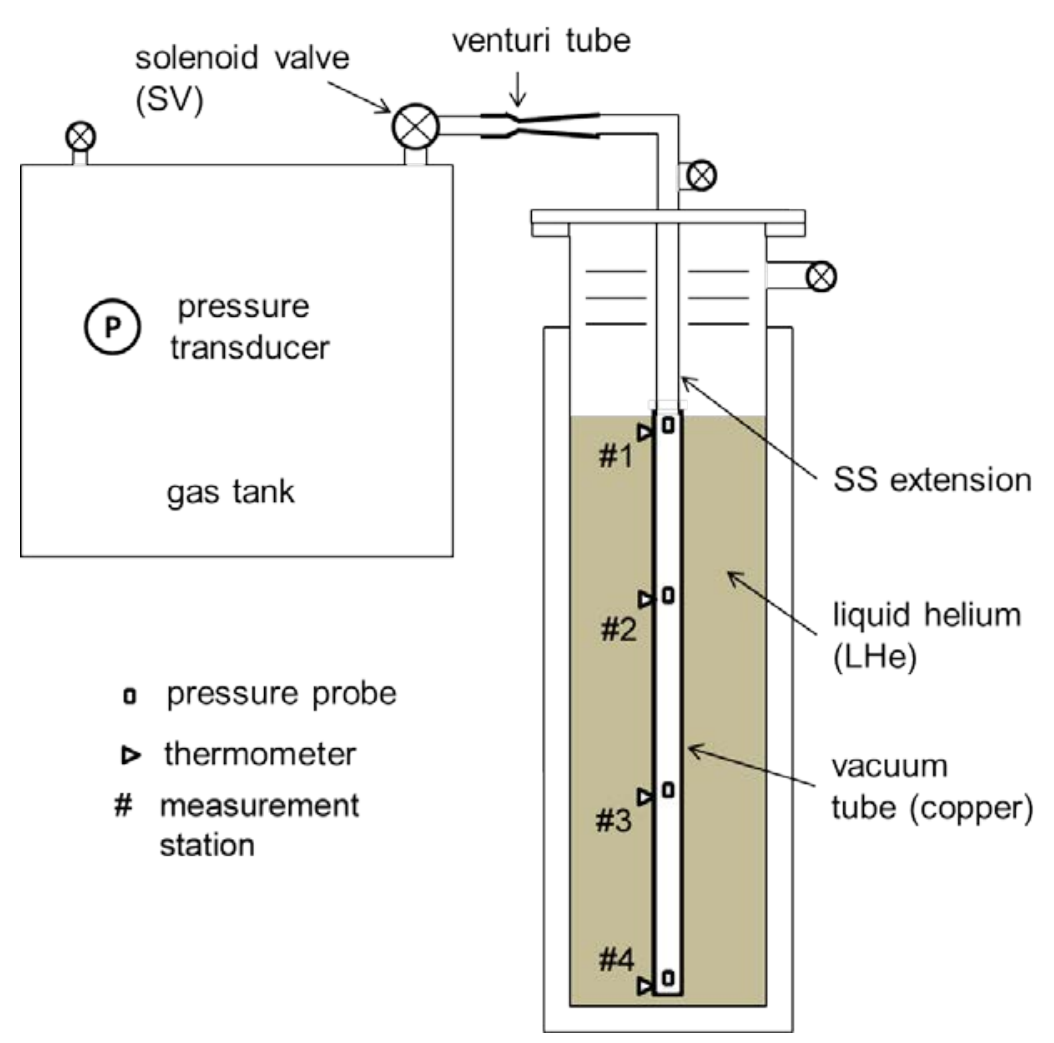

Figure 1: Schematic of the propagation measurement setup.

experiments a $3 \mu \mathrm{A}$ constant current source (in-house design) powered the thermometers while the voltage drop was recorded by Data Translation DT-9824 DAQs at $4.8 \mathrm{kHz}$ per sensor. Temperature measurement uncertainty is typically $\pm 20 \mathrm{mK}$ in the $2-20 \mathrm{~K}$ range and $\pm 0.2 \mathrm{~K}$ above $20 \mathrm{~K}$. All DAQ boards are time-referenced to the voltage trigger that opens the solenoid valve. This trigger also provides the 'zero time' for all the measurements.

\section{Results and Discussion}

We first present and discuss the results from the loss-of-vacuum experiments that started with the vacuum tube at three different temperatures- $295 \mathrm{~K}, 77 \mathrm{~K}$, and $4.2 \mathrm{~K}$. By introducing an increasing temperature difference between the gas flowing in (at room temperature) and the tube wall, these experiments signify the effect of the heat and mass transfer on the gas propagation speed. The gas front propagation in the LHe cooled tube is then described in terms of the pressure and temperature data recorded during the $4.2 \mathrm{~K}$ experiment. The propagation speed is determined by analyzing these data in a later section of this paper.

\section{a) Effect of heat and mass transfer on propagation speed}

Separate experiments with the vacuum tube at $295 \mathrm{~K}, 77 \mathrm{~K}$, and $4.2 \mathrm{~K}$ bring out the effect of the heat and mass transfer from the gas to the tube, on the gas propagation speed. Each experiment started with $100 \mathrm{kPa}, 295 \mathrm{~K}$ nitrogen gas (99.999\% pure) in the supply tank while the tube was evacuated to $\approx 10^{-4} \mathrm{~Pa}$. Figure 2(a) shows the propagation captured by the four pressure probes, with $0.5 \mathrm{~m}$ spacing, during an experiment with the tube at room temperature ( $\mathrm{RT} \approx 295 \mathrm{~K}$; the experiment referred to as 'run-RT'). Travel time of $\approx 2.5 \mathrm{~ms}$ over $1.5 \mathrm{~m}$ (first and fourth probe) gives an average propagation speed of 
$\approx 600 \mathrm{~m} / \mathrm{s}$. In another experiment the vacuum tube was immersed in liquid nitrogen, which holds the tube wall at $77 \mathrm{~K}$ (run-LN2). In figure 2(b), the pressure traces from run-LN2 also show the speed to be $\approx 600 \mathrm{~m} / \mathrm{s}$. Next, the evacuated tube was cooled by liquid helium at $4.2 \mathrm{~K}$ (run-LHe). The pressure traces after the loss of vacuum in this experiment (figure 2(c)) show drastic reduction in the propagation speed. A travel time of $\approx 450 \mathrm{~ms}$ over $1.5 \mathrm{~m}$ translates to an average propagation speed of $\approx 3.3 \mathrm{~m} / \mathrm{s}$.

In run-RT the tube wall at $295 \mathrm{~K}$ cannot condense nitrogen gas. Assuming the dynamics of expansion does not produce a large drop in the gas temperature, the convective heat exchange between the gas ( $\approx 295 \mathrm{~K})$ and the $295 \mathrm{~K}$ tube is expected to be low. In run-LN2 convective heat exchange between the gas and the $77 \mathrm{~K}$ tube will be greater than that in run-RT. Condensation is still not possible because the pressure in the tube $(<0.5 \mathrm{kPa})$ for the duration of propagation $(0-3 \mathrm{~ms})$ is much less than $100 \mathrm{kPa}$ - the saturation vapor pressure of nitrogen at $77 \mathrm{~K}$. Nitrogen gas pressure must be at least $100 \mathrm{kPa}$ for the gas to condense on a $77 \mathrm{~K}$ surface. Very similar propagation speeds in run-RT and run-LN2 suggest that only convective heat exchange between the gas and the tube wall is insufficient to slow the propagation. In

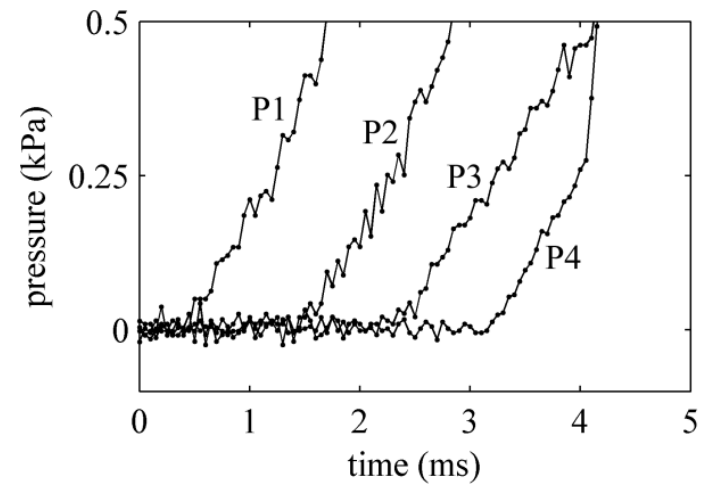

(a)

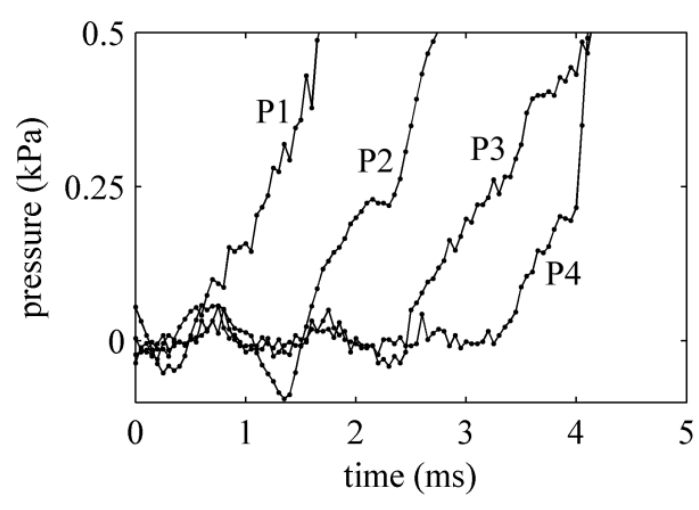

(b)

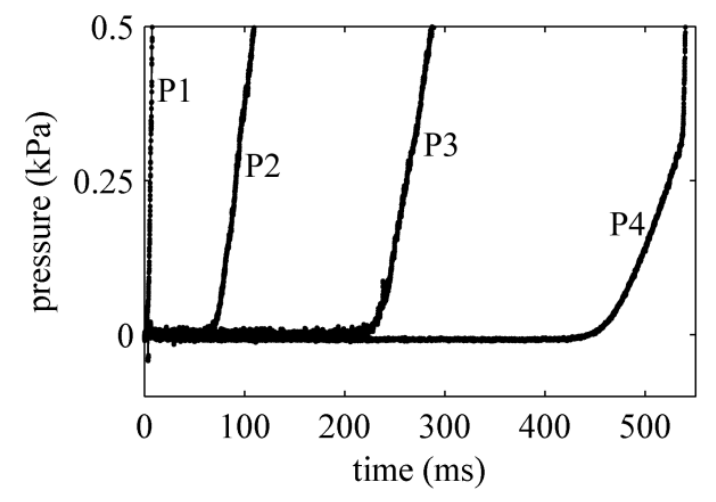

(c)

Figure 2: Pressure traces recorded at stations 1-4 during experiments with the tube at (a) room temperature (run-RT) (b) $77 \mathrm{~K}$ (run-LN2) (c) $4.2 \mathrm{~K}$ (run-LHe). All experiments started with $100 \mathrm{kPa}$, $295 \mathrm{~K}$ nitrogen gas in the supply tank and the vacuum tube at $\approx 10^{-4} \mathrm{~Pa}$ 
run-LHe the tube wall is at $4.2 \mathrm{~K}$ and the corresponding saturation vapor pressure of nitrogen is $<<10^{-10}$ $\mathrm{Pa}$ [8]. Due to such low saturation vapor pressure nitrogen gas will extensively condense (solidify) on the tube inner surface immediately after entering the tube. A much smaller mass will remain as gas and propagate forward with a decreased speed. Based on these observations, we conclude that the tube temperature must be low enough to condense the gas so as to slow its propagation.

\section{b) The run-LHe pressure and temperature data}

The run-LHe pressure and temperature traces recorded at stations 1-4 in the tube are displayed in figure 3(a) and figure 3(b) respectively. The process of pressurization (figure 3(a)) can be separated into two regimes. Regime 1 consists of a forward propagating pressure front, which sets in immediately after the vacuum loss. The pressure rise at station 1 is detected by the pressure probe $<5 \mathrm{~ms}$ after opening the $\mathrm{SV}$, indicating the arrival of the pressure front at this station. After a sharp increase, the pressure at station 1 saturates because the incoming gas is drawn into the cold vacuum space downstream. The pressure front passes sequentially through stations 2-4 raising the pressure in the entire tube. Condensation of this traveling pressure front on the tube heats the tube gradually along its length. The temperature traces in figure 3(b) show this process, which can be pictured as a heat wave propagating in the tube wall (see Theoretical Considerations for a detailed illustration). For the data in figure 3, the forward propagation lasts for about $0.5 \mathrm{~s}$. In longer channels viz. accelerator beam-lines, the forward propagation will last even longer. The speed of forward propagating pressure front is of particular significance as this speed will dictate the allowable time one has to arrest this front over a given length (a critical safety consideration in accelerator design). In this paper, we will focus on the forward propagation of the pressure front.

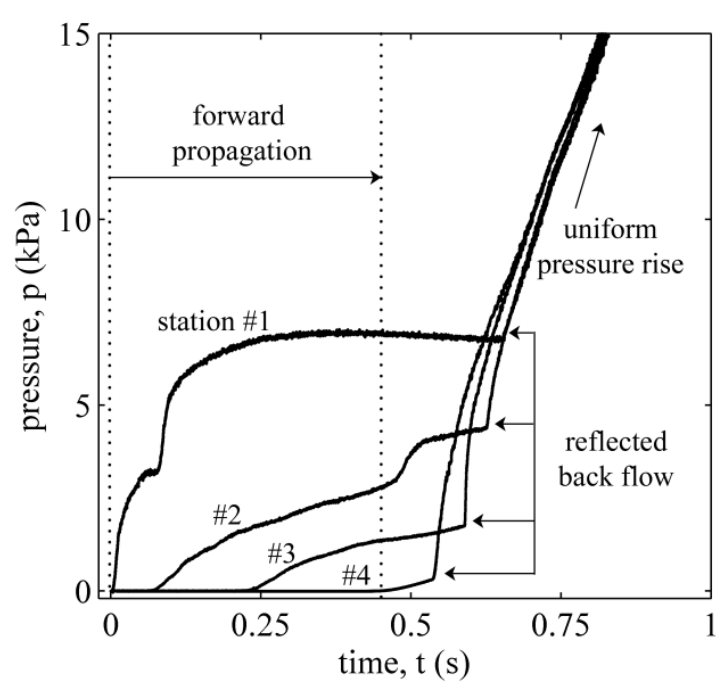

(a)

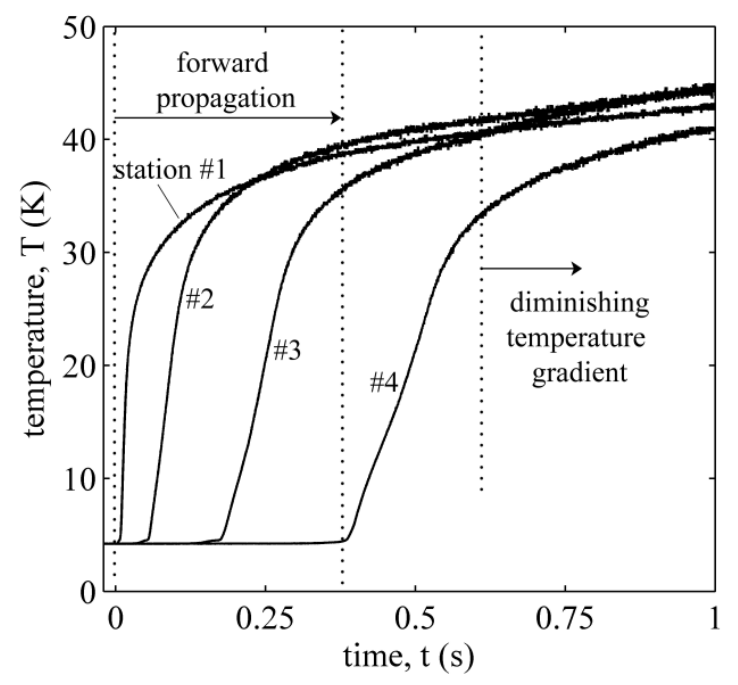

(b)

Figure 3: (a) Tube pressure and (b) tube temperature recorded at stations 1-4 in the tube after the loss of vacuum. The sensors capture the forward propagation of the pressure front followed by spatially uniform pressure and temperature rise. 
Upon crossing station 4 the gas front encounters the closed end of the tube. The pressure traces in figure 3(a) depict the flow of gas that reflects from the closed end. Unable to propagate forward due to the closed end, the gas further flowing in pressurizes the tube uniformly over its length. In this regime (regime 2), the gas condenses uniformly over the tube. Consequently the tube temperature gradient (figure 3(b)), which was significant during the forward propagation, starts to diminish. Eventually, the tube pressurizes to atmosphere and attains a steady temperature (time $>1 \mathrm{~s}$, not shown in figure 3(b)). The tube being considerably warmer than the $4.2 \mathrm{~K}$ LHe bath will now transfer large heat fluxes to the helium (another concern in the safety of accelerators). Further analysis of this regime and the estimates of LHe heat load are presented in [9].

\section{c) Comparing the front propagation recorded by the pressure probes and the thermometers}

The speed of front propagation can be obtained from the front arrival times recorded by the pressure probes as well as the thermometers. Figure 4 compares the front propagation in run-LHe recorded by the pressure probes (top) and the thermometers (bottom). Note that the axial locations of the pressure probes inside the tube exactly match those of the thermometers on the tube wall. Two interesting observations from figure 4 are noticed: first, at all locations except the tube entrance, the thermometer shows a temperature rise before the corresponding pressure probe detects rise in pressure. This early temperature rise is because condensation at any location in the tube will begin at nitrogen gas pressure $<10^{-10} \mathrm{~Pa}$, which is too low to be detected by the pressure probe. Due to condensation heat transfer to the tube, the tube temperature at a location will already have risen above $4.2 \mathrm{~K}$ well before the pressure level at that location becomes readable. The thermometers thus capture the gas arrival times more precisely than the pressure probes. We will therefore obtain the propagation speed in terms of the arrival times recorded by the thermometers. Second, the increasing travel times between successive thermometers indicate deceleration of the pressure front along the tube. In a later section of this paper we will develop a

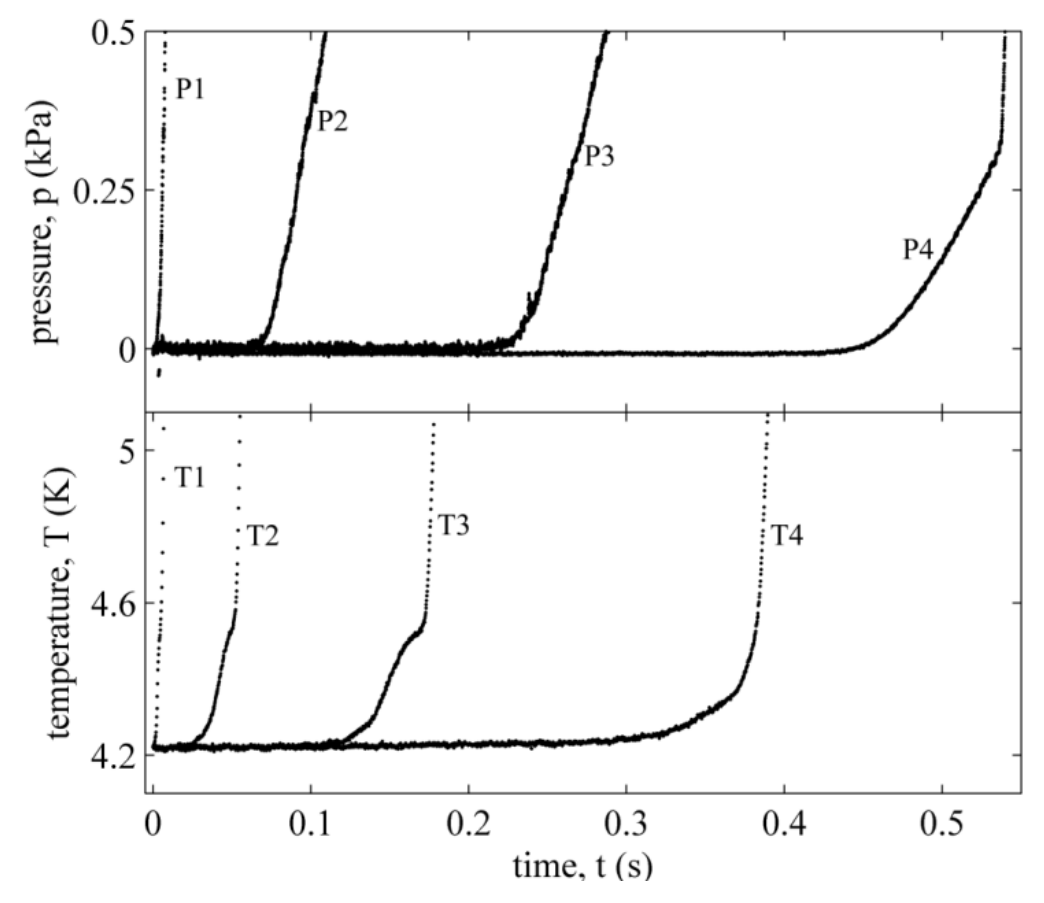

Figure 4: Comparison of the run-LHe pressure and temperature traces. 
theoretical framework that accounts for this deceleration.

To further explore the front deceleration, eight more thermometers were mounted on the vacuum tube to measure the arrival times at twelve locations along the tube. With this enhancement, the successive thermometers have $0.125 \mathrm{~m}$ spacing except the last pair, where the two thermometers are spaced by $0.25 \mathrm{~m}$. Additionally, loss-of-vacuum experiments were conducted with different mass in-flow rates of nitrogen gas to verify that the front deceleration is not an artifact of a particular mass in-flow rate. Three different rates of nitrogen mass-flow into the tube were generated in separate experiments by starting with the gas at $50 \mathrm{kPa}, 100 \mathrm{kPa}$, and $150 \mathrm{kPa}$ in the supply tank. These pressures respectively yielded near constant mass flow rates of $8.1 \mathrm{~g} / \mathrm{s}, 18.3 \mathrm{~g} / \mathrm{s}$, and $27.7 \mathrm{~g} / \mathrm{s}$ (the procedure to calculate the mass flow rate is described in Appendix). These flow rates were chosen arbitrarily, but yielded noticeably different

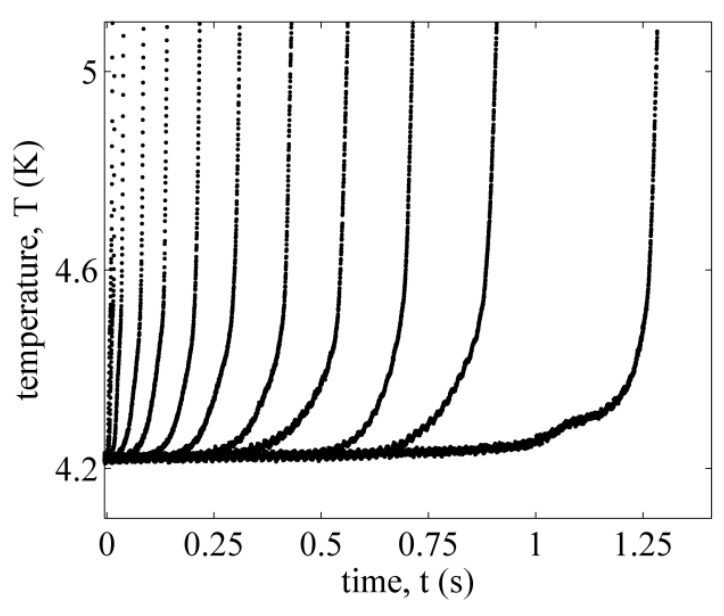

(a)

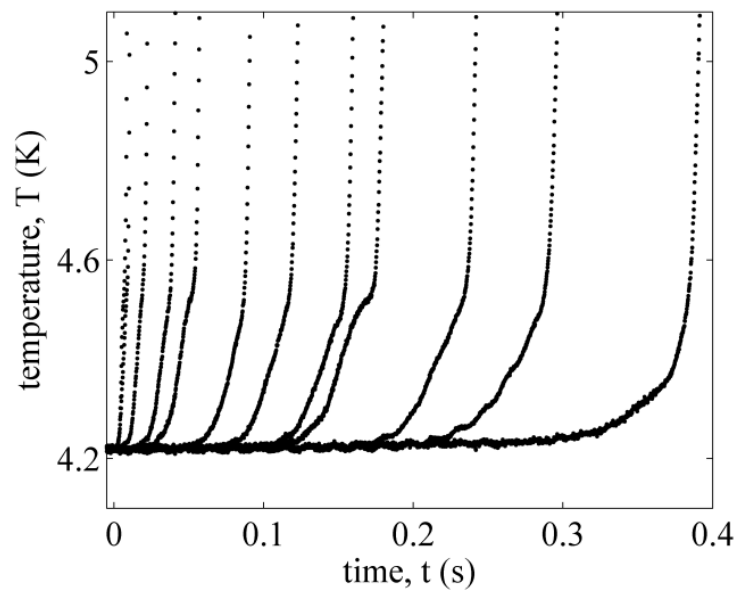

(b)

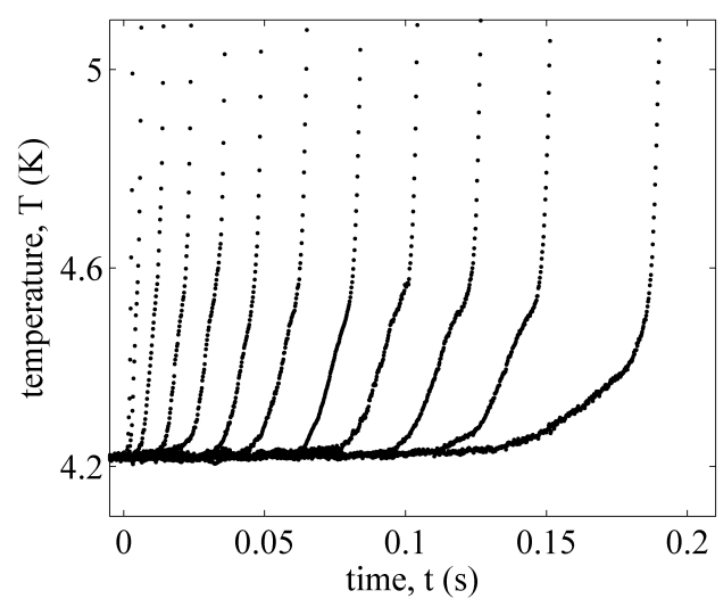

(c)

Figure 5: Temperature traces recorded by twelve thermometers on the tube during experiments starting with (a) $50 \mathrm{kPa}$ (b) $100 \mathrm{kPa}$, and (c) $150 \mathrm{kPa}$ nitrogen gas in the supply tank. The thermometers are spaced by $0.125 \mathrm{~m}$ except the last pair, which has $0.25 \mathrm{~m}$ spacing. 
propagation speeds to allow for a comparison. Figure 5 depicts the temperature traces recorded during these experiments. The front travel times are $\approx 1 \mathrm{~s}(50 \mathrm{kPa}$, figure $5(\mathrm{a})), \approx 0.3 \mathrm{~s}(100 \mathrm{kPa}$, figure 5(b)), and $\approx 0.13 \mathrm{~s}(150 \mathrm{kPa}$, figure 5 (c)) over a length of $1.5 \mathrm{~m}$. For all the three mass in-flow rates the front is seen to decelerate along the tube. In a later section, we will calculate the propagation speed and its deceleration from the data of figure 5 .

The temperature traces in figure 5 show that lower mass in-flow rate will result in slower propagation. In another experiment, the venturi tube (see figure 1 for the location of the venturi tube) was replaced by a small orifice to obtain even smaller mass flow rates from the supply tank. On opening the solenoid valve, this orifice slowly bled the gas in the supply tank to the vacuum tube at an arbitrary near-constant mass flow rate of $0.15 \mathrm{~g} / \mathrm{s}$. Figure 6 displays the tube temperature measured by four thermometers (with $0.5 \mathrm{~m}$ spacing) in this experiment. Only the first thermometer, which is located at the entrance of the cold tube, shows rise in temperature. All other thermometers located downstream in the tube stay put at $4.2 \mathrm{~K}$. At such low mass in-flow rates, all the incoming gas steadily condenses near the entrance of the LHe cooled section of the tube. Note that in this experiment, the solenoid valve was kept open for as many as $20 \mathrm{~s}$ with the anticipation of a very slow propagating pressure front. A propagating front, however, was not observed. This case of low mass flow rate resulting in a vanishing front propagation is not further analyzed in this paper.

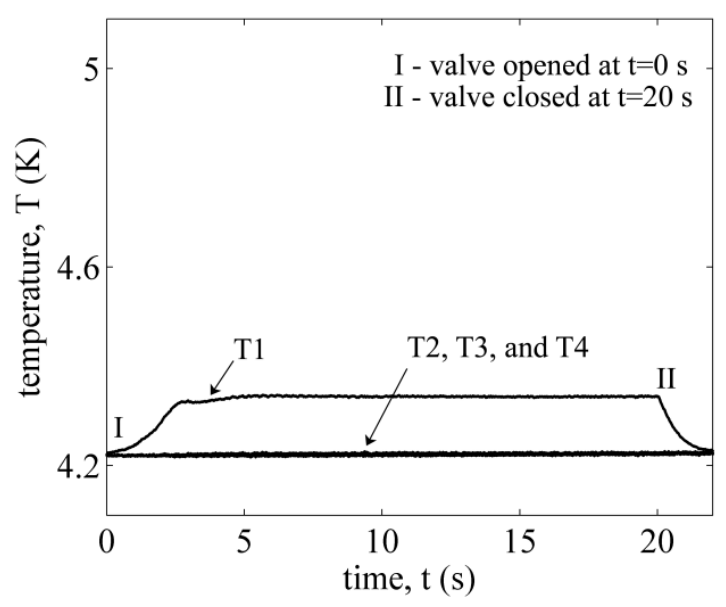

Figure 6: Tube temperature traces in an experiment with $0.15 \mathrm{~g} / \mathrm{s}$ in-flow rate of nitrogen.

\section{Theoretical considerations}

The objective here is to gain a general understanding of the experimental observations. To do so, we obtain an analytical expression for the front propagation speed in a vacuum tube in presence of condensation by applying conservation of mass to the situation depicted in figure 7 (the expanded derivation is presented in Appendix). Figure 7(a) depicts the state at time $\tau=0$ when the cold vacuum channel suddenly ruptures at $X=0$ and a condensable gas flows in to the channel at a constant (choked) flow rate $\dot{m}_{i n}$. The state at time $\tau=t$ after the rupture is schematized in figure 7(b). At this time the gas front is located at $X=x$ (i.e., it has traversed a length $x$ along the channel), the gas in the region $(0, x)$ 
exhibits density $\rho(X, t)$, and condenses on the channel inner surface at a rate $\dot{m}_{d e p}^{\prime \prime}(X, t)$ per unit surface area.

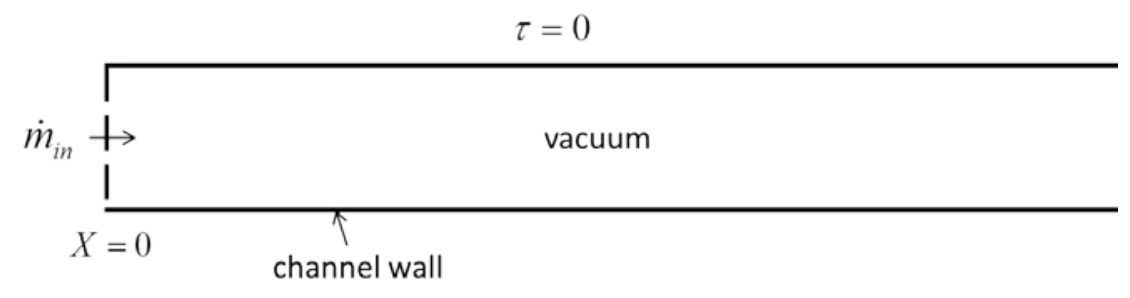

(a)

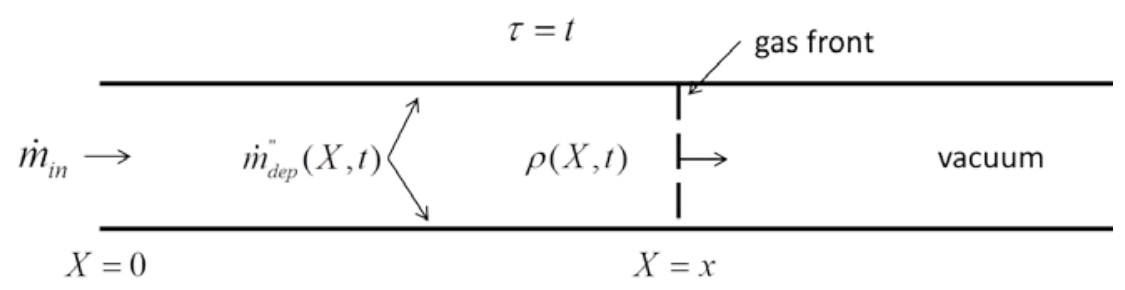

(b)

Figure 7: Gas front propagation in a vacuum tube in presence of condensation (a) the gas enters the vacuum tube at $X=0$ and the front propagates down the vacuum space (b) at time $\tau=t$ the front is located at $X=X$, the density in the region occupied by the gas is $\rho(X, t)$, and the rate of condensation (per unit surface of area of the channel) is $\dot{m}_{d e p}^{\prime \prime}(X, t)$.

Conservation of mass in the scenario of figure 7(b) dictates that the mass of gas entering the tube must divide into three components- the mass that accumulates as gas in the region $(0, x)$, the mass that condenses on the channel wall, and the mass that is carried by the gas front. Mathematically, this condition can be written down on a per unit time basis as:

$\dot{m}_{i n}=\frac{\pi D^{2}}{4} \int_{0}^{x} \frac{\partial \rho(X, t)}{\partial t} d X+\pi D \int_{0}^{x} \dot{m}_{d e p}^{\prime \prime}(X, t) d X+\left.\left.\frac{\pi D^{2}}{4} \rho\right|_{x} v\right|_{x}$

where $\left.\rho\right|_{x}$ and $\left.v\right|_{x}$ respectively are the density and velocity at the gas front. Rearranging equation 1 yields an explicit expression for the front propagation speed:

$$
\left.v\right|_{x}=\frac{\dot{m}_{i n}-\frac{\pi D^{2}}{4} \int_{0}^{x} \frac{\partial \rho(X, t)}{\partial t} d X-\pi D \int_{0}^{x} \dot{m}_{d e p}^{\prime \prime}(X, t) d X}{\left.\frac{\pi D^{2}}{4} \rho\right|_{x}}
$$

The propagation speed at $X=x$, according to equation 2, depends on the rate of change of density and the rate of mass condensation over the length $x$. Clearly, the mass deposition term in equation 2 will 
result in slower propagation compared to the room temperature or even the $\mathrm{LN}_{2}$ case wherein $\dot{m}_{d e p}^{\prime \prime}$ is essentially zero. When $\dot{m}_{d e p}^{\prime \prime}$ is zero the propagation speed will depend only on the integral of the rate of change of density. Practically constant propagation speeds in the channel at room temperature (figure 2(a)) and when immersed in $77 \mathrm{~K} \mathrm{LN}_{2}$ (figure 2(b)) indicate that the density integral term should be small compared to the mass in-flow rate. For the $4.2 \mathrm{~K}$ LHe case, the density integral term is evaluated approximately using the tube pressure data (see Appendix) and is also found to be small compared to the mass in-flow rate. Neglecting the density integral term equation 2 reduces to:

$$
\left.v\right|_{x}=\frac{\dot{m}_{i n}-\pi D \int_{0}^{x} \dot{m}_{d e p}^{\prime \prime}(X, t) d X}{\left.\frac{\pi D^{2}}{4} \rho\right|_{x}}
$$

so that the propagation speed depends on the rate of mass condensation over the length $x$ of the channel. The length of the channel condensing the gas gets longer as the front travels further in the channel. The increasing channel surface area $(\pi D x)$ will condense an increasing portion of the constant in-flux of the gas and will decelerate the front as the front moves forward. Even though equation 3 qualitatively supports the decrease in front speed along the vacuum, the expression cannot be used to quantify the propagation speed. This limitation is due to the difficulties in obtaining an analytical or an empirical form of $\dot{m}_{d e p}^{\prime \prime}[10]$. We now discuss some of the complexities in determining $\dot{m}_{d e p}^{\prime \prime}$ in the context of the propagation problem.

Figure 8 displays a gas front propagating in a vacuum channel that has its outer surface wetted by LHe. Consider the gas to be nitrogen. The gas front and its wake deposit mass and heat on to the channel, which subsequently transfers heat to the adjacent LHe. The gas condensation rate at any location in the channel depends on the temperature of the condensing surface at that location, as well as the gas pressure (in order to condense, the gas pressure must exceed the saturation pressure at the temperature of the condensing surface). For the gas near the front, the condensation surface is the channel wall at $4.2 \mathrm{~K}$. The gas adjacent to the front has pressure below the triple point pressure of $12.5 \mathrm{kPa}$ (see figure 3(a)) and so this gas condenses to a solid (solid nitrogen or $\mathrm{SN}_{2}$ ). Along its path, the propagating front will thus coat the channel with $\mathrm{SN}_{2}$. The gas in the wake of the front then condenses on this $\mathrm{SN}_{2}$ layer. For the propagation data shown in figure 3 , the pressure in the wake is still less than $12.5 \mathrm{kPa}$ and so this gas will continue to form $\mathrm{SN}_{2}$. The condensation rate in the wake, however, is controlled by the temperature of the top surface of the $\mathrm{SN}_{2}$ layer. According to Fourier's law of heat conduction, the $\mathrm{SN}_{2}$ top surface 


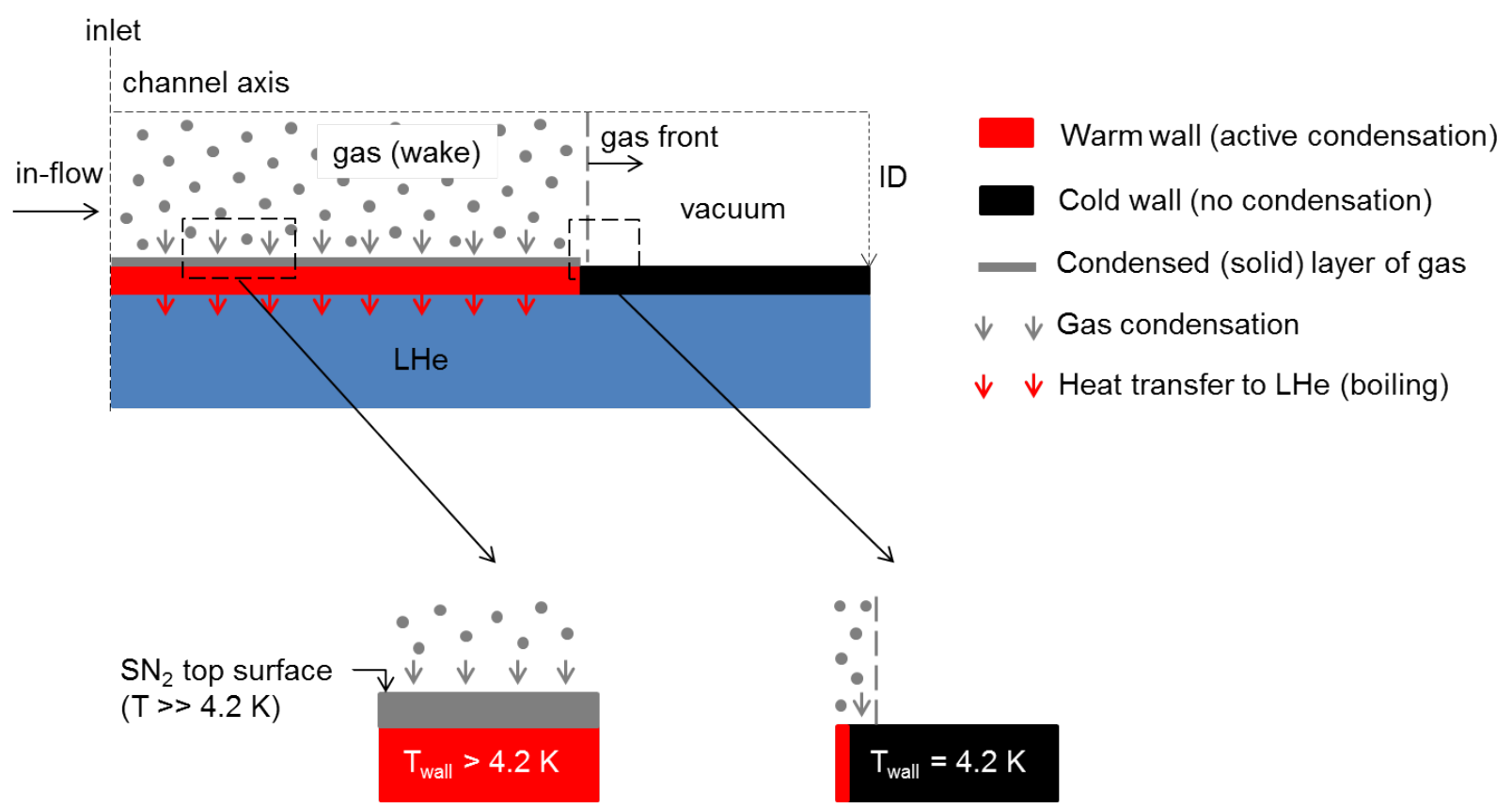

Figure 8: Condensation heat and mass transfer to the channel and the subsequent heat flow into the LHe. The gas adjacent to the front condenses on the $4.2 \mathrm{~K}$ channel while the gas far behind the front condenses on a layer of solid nitrogen $\left(\mathrm{SN}_{2}\right)$.

temperature will be a function of the heat diffusing through the solid layer, the layer's thermal resistance (thickness/thermal conductivity), and the channel wall temperature. All these parameters vary with time and location in the channel, and with the possible exception of the channel wall temperature, are difficult to measure. Specifically, the $\mathrm{SN}_{2}$ layer thickness (and so its thermal resistance) will grow with time and at a given time will also vary along the channel- the layer near the inlet may be thicker than further down in the channel. Due to these highly dynamic processes of heat and mass transfer, the $\mathrm{SN}_{2}$ surface temperature and the condensation rate on this layer are extremely difficult to quantify (also see [10]).

\section{Empirical formulation of the propagation speed}

An empirical functional form for the front propagation speed is obtained from the data in figure 5 to describe the front deceleration along the tube. The front arrival times needed to obtain this functional form are extracted from the temperature traces of figure 5 by a procedure described further. The arrival time at a given location is defined as the time when the temperature at that location rises from its initial value (background) to a certain threshold. Note that the temperature data in figure 5 are corrupted by electromagnetic interference or noise, and the temperature threshold therefore needs a statistical definition.

Figure 9 shows a typical temperature vs. time trace carrying $60 \mathrm{~Hz}, 20 \mathrm{mK}$ peak-to-peak electromagnetic interference. The arrival time is determined from such a noisy trace using the following procedure:

1) Calculate mean $(\mu)$ and standard deviation $(\sigma)$ of the noisy temperature data for time $t<0$ (i.e. before loss of vacuum). 4800 data points (data in the time interval -1 to 0 s) are used for calculating $\mu$ and $\sigma$. The trace in figure 9 has $\mu=4.22 \mathrm{~K}$ and $\sigma=3.4 \mathrm{mK}$. 
2) Define threshold as $\mu+3 \sigma$ so that the threshold is greater than the values of $99.7 \%$ of the background $(\mathrm{t}<0)$ data. Threshold for the data in figure 9 is $4.23 \mathrm{~K}$.

3) Run a moving average filter across the noisy data to determine the local mean. The window size ' $w$ ' of this moving average is chosen according to the rate of temperature rise- a narrow window size (11 samples) for sharp rise in temperature, e.g. at locations very near the vacuum tube entrance (see figure 4); a wider window size- maximum 81 samples where temperature rise is gradual (window size of 81 will perfectly smooth the $60 \mathrm{~Hz}$ noise in the data sampled at $4800 \mathrm{~Hz}$ ). The moving average in figure 9 uses a window size of 61 samples.

4) Define arrival time as the time when the moving average (local mean) equals the threshold. The maximum uncertainty in this rise time is the width of moving average window (6 ms for the moving average in figure 9).

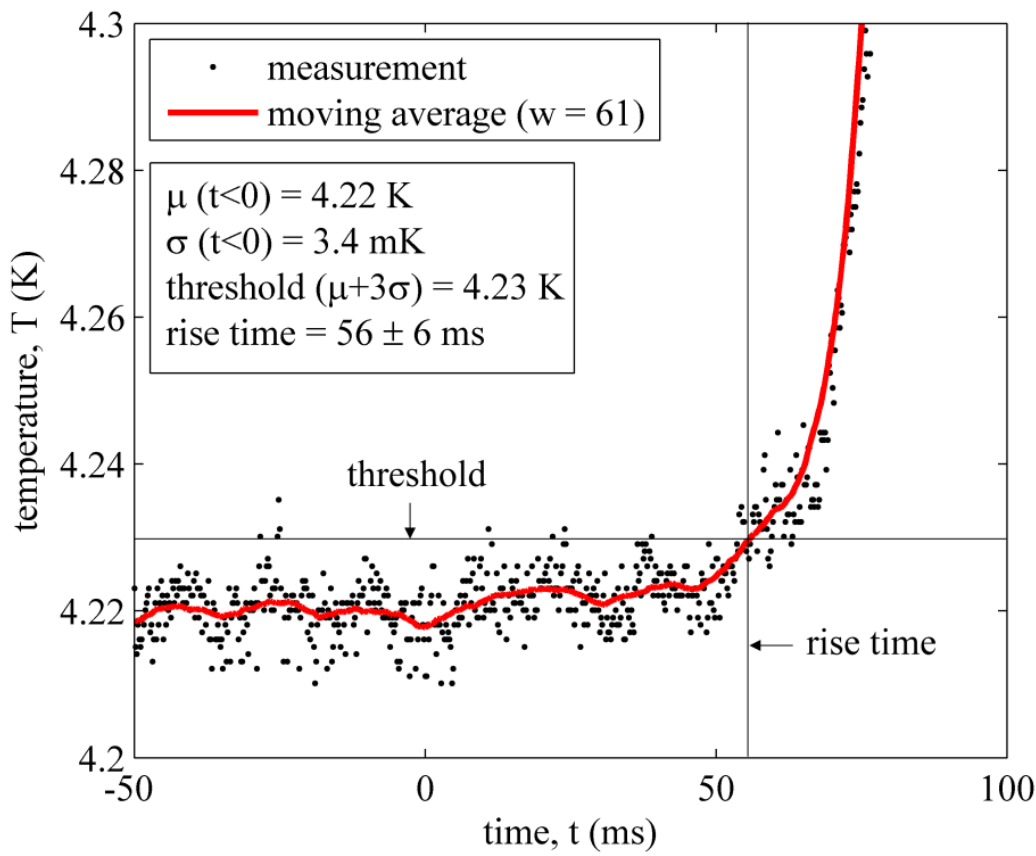

Figure 9: Determining arrival time from a noisy trace using a statistical definition of threshold temperature.

Figure 10 shows the arrival times at twelve locations in the vacuum tube for experiments with the three starting pressures of $50 \mathrm{kPa}, 100 \mathrm{kPa}$, and $150 \mathrm{kPa}$. The corresponding mass in-flow rates are $8.1 \mathrm{~g} / \mathrm{s}$, $18.3 \mathrm{~g} / \mathrm{s}$, and $27.7 \mathrm{~g} / \mathrm{s}$ respectively. The error bars on the rise times correspond to the uncertainty introduced by the moving average method (maximum uncertainty is $\pm 8 \mathrm{~ms}$ for a moving average window of 81 samples). Also plotted in figure 10 are the two-parameter exponential fits $t=a\left(e^{x / b}-1\right)$, which are found to best represent the arrival time-location data ( $t$ is the arrival time at location $x$ ). The fit coefficients $a$ and $b$ are obtained by non-linear least squares regression using the method of differential corrections [11] and are tabulated in figure 10 along with their standard errors. 


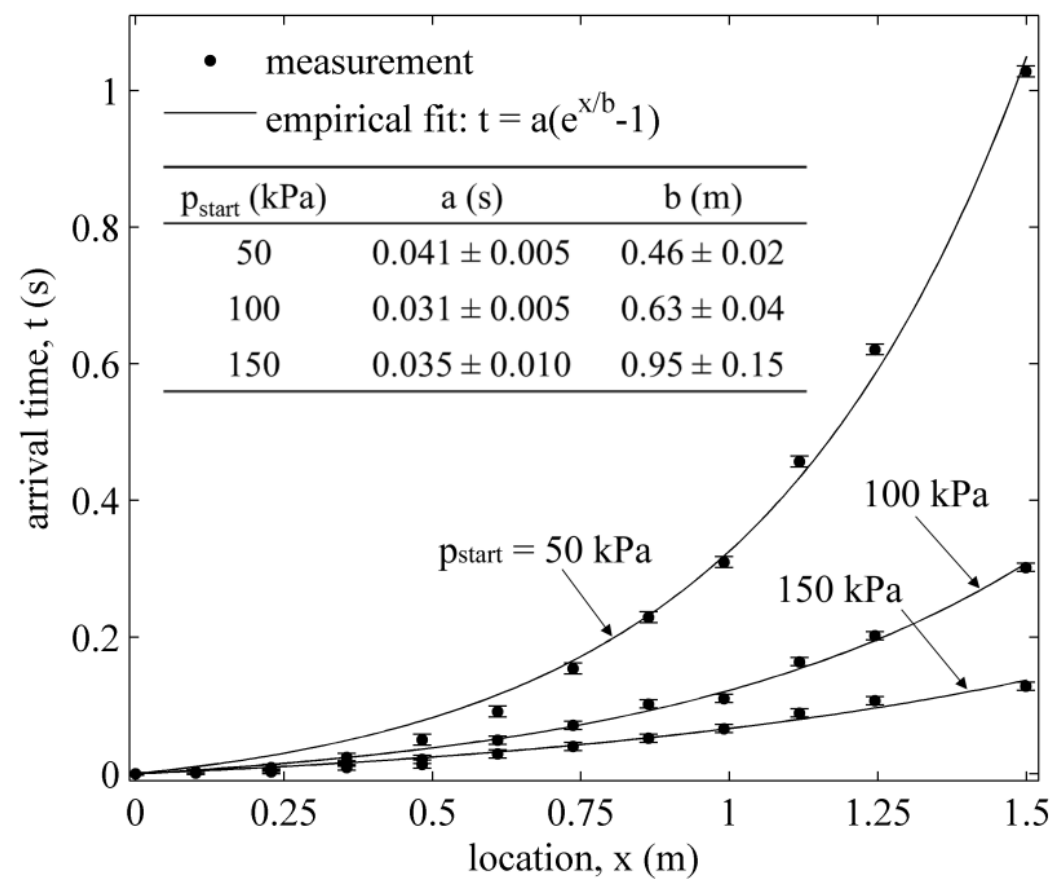

Figure 10: Front arrival time vs. location for experiments starting with $50 \mathrm{kPa}, 100 \mathrm{kPa}$, and $150 \mathrm{kPa}$ nitrogen gas in the supply tank. A two parameter empirical model $t=a\left(e^{x / b}-1\right)$ is fit to the data.

The arrival time-location fit yields propagation speed $v=v_{0} e^{-x / b}$, which decreases exponentially along the tube. In this expression $v_{0}=b / a$ is the speed at the first thermometer station (start of the condensing section of the tube) and $b$ is the travel length over which the speed falls to $v_{0} / e$. Although the exponential fits represent the data from the three experiments reasonably well, the physics governing this exponential decay is less clear at present and is a subject of further study.

\section{Conclusions}

The experimental study reported in this paper identifies gas condensation as the criteria necessary for the slow propagation of a gas in a vacuum tube. When approached analytically, we found that the front propagation speed at a given time and location in the tube is controlled by the gas condensation rate over the length that the front has traveled till this time. This length continuously increases as the front propagates in the tube. The front deceleration along the tube is attributed to the continuously increasing surface area of the tube that the gas condenses on. This increasing surface area removes more and more gas from the region behind the gas front and slows its propagation. Our analytical model could not quantify the propagation speed because the mass condensation rates on to the tube are largely unknown. Analysis of the measured arrival time data yielded propagation speed that decreases exponentially along the tube. 


\section{Acknowledgement}

Research supported by US Department of Energy Grant DE-FG02-96ER40952. We sincerely thank Prof. Wei Guo, Department of Mechanical Engineering and Prof. David Kopriva, Department of Mathematics at Florida State University for several discussions in the course of experiments and analysis.

\section{Appendix}

\section{A1. Calculation of the mass flow rate into the vacuum tube}

Mass in-flow rates, nearly constant for the duration of propagation, were generated by installing a venturi tube at the supply tank outlet. In this configuration, the flow chokes at the throat of the venturi due to a large pressure difference across its inlet (tank pressure) and its outlet (vacuum tube pressure). Choking is verified by observing a relation $p^{b} \propto t$ between the tank pressure $p$ and time $t$ [12] $(b=(1-\gamma) / 2 \gamma$ and $\gamma=1.4$, the ratio of specific heat capacities for nitrogen). An example of choked flow is shown in figure A1 (top), in which a $p^{b} \propto t$ fit accurately follows the tank pressure - time trace recorded during the experiment that started with $100 \mathrm{kPa}$ nitrogen gas. Clearly, choking persists for times beyond the duration of propagation $(0-350 \mathrm{~ms})$. The choked mass in-flow rate, assuming isentropic gas dynamics in the supply tank, is [12]:

$\dot{m}_{\text {in }}=-\left(\frac{p_{\text {start }}^{(\gamma-1) / \gamma} V}{\gamma R T_{\text {start }}}\right)\left(p^{-(\gamma-1) / \gamma} \frac{d p}{d t}\right)$

where $p$ is the supply pressure and $V$ is the tank volume (85 liters). $p_{\text {start }}$ and $T_{\text {start }}$ respectively are the starting gas pressure and temperature $(295 \mathrm{~K})$, and $R$ is the gas constant ( $297 \mathrm{~J} / \mathrm{kg}-\mathrm{K}$ for nitrogen). Figure A1 (bottom) depicts the mass in-flow rate generated with $100 \mathrm{kPa}$ nitrogen gas. Although choked, the fall in the mass flow rate is due to the decreasing supply pressure. To account for this decrease, we use

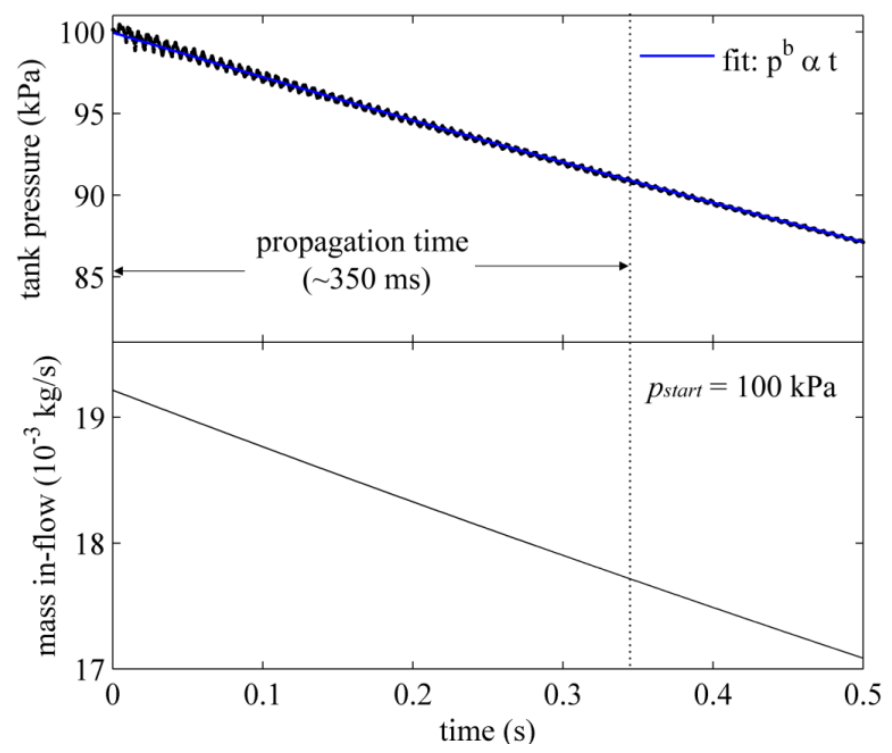

Figure A1: Choked mass in-flow rate for the $100 \mathrm{kPa}$ run_LHe. 
Table A1: Mass flow rates obtained with different starting pressures

\begin{tabular}{cccc}
\hline \multirow{2}{*}{$p_{\text {start }}(\mathrm{kPa})$} & \multicolumn{2}{c}{$\dot{m}_{\text {in }}\left(10^{-3} \mathrm{~kg} / \mathrm{s}\right)$} & Average $\dot{m}_{\text {in }}\left(10^{-3} \mathrm{~kg} / \mathrm{s}\right)$ \\
\cline { 2 - 3 } & start $^{1}$ & end $^{2}$ & 8.1 \\
50 & 9.2 & 7.4 & 18.3 \\
100 & 19.1 & 17.6 & 27.7 \\
150 & 28.4 & 27 & $\mathrm{kPa}-130 \mathrm{~ms}$
\end{tabular}

time averaged in-flow rates. Table A1 lists the mass in-flow rates at start and end of the forward propagation, and the average values of mass in-flow rates over this duration. The uncertainty in mass flow rate measurement in all the experiments is $\pm 0.1 \mathrm{~g} / \mathrm{s}$.

\section{A2. Derivation of the expression for propagation speed}

Here, we formulate an expression for the front propagation using first principles to identify the cause of the slow propagation and of the deceleration of the gas front as revealed by the experiments. To derive an expression for the front propagation speed we analyze the scenario in figure A2.1, which depicts gas propagation in a vacuum channel in presence of condensation. Following a loss of vacuum at time $\tau=0$, the gas enters the channel at location $X=0$ at a constant in-flow rate of $\dot{m}_{\text {in }}$ (figure A2.1 (a)), and a

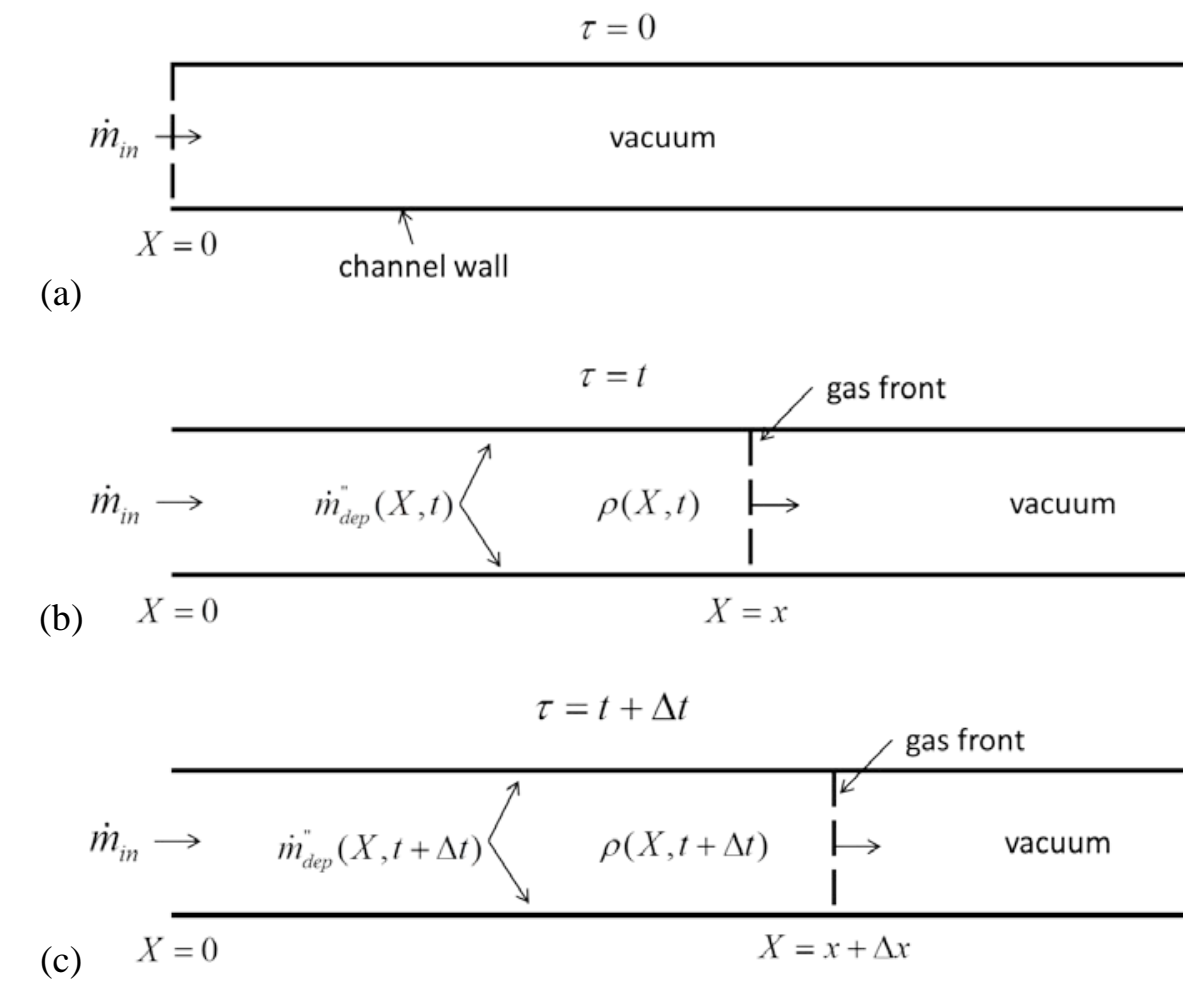

Figure A2.1: Schematic of gas flow in a vacuum channel in presence of condensation (a) time $\tau=0$, the channel ruptures allowing the gas to enter at $X=0$ (b) time $\tau=t$, the gas front is at $X=x$ (c) time $\tau=t+\Delta t$, the gas front is at $X=x+\Delta x$. 
pressure front propagates along $+X$ direction. At time $\tau=t$ the gas in the channel exhibits density $\rho(X, t)$ and condenses on the channel at a rate $\dot{m}_{d e p}^{\prime \prime}(X, t)$ per unit surface area of the channel wall. At $\tau=t$ the gas front has traveled to $X=x$ (figure A2.1(b)) while at $\tau=t+\Delta t$ the gas front, having traveled an extra length $\Delta x$, is located at $X=x+\Delta x$ (figure A2.1(c)). The front propagation speed is then defined as $\Delta x / \Delta t$ and is derived by applying conservation of mass in the following way- from time $\tau=0$ to $\tau=t$ :

$\dot{m}_{i n} *(t-0)=\frac{\pi D^{2}}{4} \int_{0}^{x} \rho(X, t) d X+\pi D \int_{0}^{x} \int_{t_{0}(X)}^{t} \dot{m}_{d e p}(X, \tau) d \tau d X$

and from $\tau=0$ to $\tau=t+\Delta t$ :

$\dot{m}_{i n} *(t+\Delta t-0)=\frac{\pi D^{2}}{4} \int_{0}^{x+\Delta x} \rho(X, t+\Delta t) d X+\pi D \int_{0}^{x+\Delta x} \int_{t_{0}(X)}^{t+\Delta t} \dot{m}_{d e p}^{\prime \prime}(X, \tau) d \tau d X$

The first term on the RHS of equations A2.1 and A2.2 represents the mass in the gaseous phase while the second term denotes the mass of gas that has condensed on the channel wall. The lower limit $t_{0}(X)$ of the time integral in the mass condensation term represents the time of onset of mass deposition (i.e., the time of arrival of the gas front) at location $X$ in the channel. Subtracting equation A2.1 from equation A2.2 yields:

$$
\dot{m}_{i n} \Delta t=\frac{\pi D^{2}}{4}\left[\int_{0}^{x+\Delta x} \rho(X, t+\Delta t) d X-\int_{0}^{x} \rho(X, t) d X\right]+\pi D\left[\int_{0}^{x+\Delta x} \int_{t_{0}(X)}^{t+\Delta t} \dot{m}_{d e p}^{\prime \prime}(X, \tau) d \tau d X-\int_{0}^{x} \int_{t_{0}(X)}^{t} \dot{m}_{d e p}^{\prime \prime}(X, \tau) d \tau d X\right]
$$

The first bracketed term (denoted by $I$ ) on the RHS of equation A2.3 is simplified in the following manner:

$$
\begin{aligned}
I & =\int_{0}^{x+\Delta x}\left[\rho(X, t)+\Delta t \frac{\partial \rho(X, t)}{\partial t}+O\left(\Delta t^{2}\right)\right] d X-\int_{0}^{x} \rho(X, t) d X \\
& =\int_{0}^{x+\Delta x} \rho(X, t) d X-\int_{0}^{x} \rho(X, t) d X+\Delta t \int_{0}^{x+\Delta x} \frac{\partial \rho(X, t)}{\partial t} d X+O\left(\Delta t^{2}\right) \\
& =\left.\Delta x \rho\right|_{x}+\Delta t\left[\int_{0}^{x} \frac{\partial \rho(X, t)}{\partial t} d X+\int_{x}^{x+\Delta x} \frac{\partial \rho(X, t)}{\partial t} d X\right]+O\left(\Delta t^{2}\right) \\
& =\left.\Delta x \rho\right|_{x}+\Delta t \int_{0}^{x} \frac{\partial \rho(X, t)}{\partial t} d X+O(\Delta x, \Delta t)+O\left(\Delta t^{2}\right)
\end{aligned}
$$


The second bracketed term on RHS of equation A2.3 (denoted by II ) simplifies as:

$$
\begin{aligned}
I I & =\left[\int_{0}^{x+\Delta x} \int_{t_{0}(X)}^{t+\Delta t} \dot{m}_{d e p}^{\prime \prime}(X, \tau) d \tau d X-\int_{0}^{x} \int_{t_{0}(X)}^{t} \dot{m}_{d e p}^{\prime \prime}(X, \tau) d \tau d X\right] \\
& =\int_{0}^{x} \int_{t_{0}(X)}^{t+\Delta t} \dot{m}_{d e p}^{\prime \prime}(X, \tau) d \tau d X+\int_{x}^{x+\Delta x} \int_{t_{0}(X)}^{t+\Delta t} \dot{m}_{d e p}^{\prime \prime}(X, \tau) d \tau d X-\int_{0}^{x} \int_{t_{0}(X)}^{t} \dot{m}_{d e p}^{\prime \prime}(X, \tau) d \tau d X \\
& =\int_{0}^{x} \int_{t}^{t+\Delta t} \dot{m}_{d e p}^{\prime \prime}(X, \tau) d \tau d X+\int_{x}^{x+\Delta x} \int_{t}^{t+\Delta t} \dot{m}_{d e p}^{\prime \prime}(X, \tau) d \tau d X \\
& =\Delta t \int_{0}^{x} \dot{m}_{d e p}^{\prime \prime}(X, t) d X+O(\Delta x, \Delta t)
\end{aligned}
$$

Note while going from A2.5.2 to A2.5.3, the lower limit of the time integral in the second term of A2.5.3 is ' $t$ ' because the onset time $t_{0}(X)$ at $X=x$ is simply $t$. Recombining the simplified $I$ and $I I$ gives:

$$
\dot{m}_{i n} \Delta t=\frac{\pi D^{2}}{4}\left[\left.\Delta x \rho\right|_{x}+\Delta t \int_{0}^{x} \frac{\partial \rho(X, t)}{\partial t} d X\right]+\pi D \Delta t \int_{0}^{x} \dot{m}_{d e p}^{\prime \prime}(X, t) d X+O(\Delta x, \Delta t)+O\left(\Delta t^{2}\right)
$$

Keeping only the first order terms and on rearranging equation A2.6, the propagation speed gets the expression:

$$
\left.v\right|_{x}=\left(\frac{\Delta x}{\Delta t}\right)_{x}=\frac{\dot{m}_{i n}-\frac{\pi D^{2}}{4} \int_{0}^{x} \frac{\partial \rho(X, t)}{\partial t} d X-\pi D \int_{0}^{x} \dot{m}_{d e p}^{\prime \prime}(X, t) d X}{\left.\frac{\pi D^{2}}{4} \rho\right|_{x}}
$$

\section{A3. Estimation of the density integral term in the propagation speed expression}

Here we evaluate the integral of the rate of change of gas density in the tube using the available experimental data. The objective is to have an estimate of this quantity so as to show that the quantity is small compared to the mass in-flow rate. To evaluate $\int_{0}^{x} \frac{\partial \rho(X, t)}{\partial t} d X$ the gas is assumed to be ideal so that $\rho=p / R T$. Two processes work to change the gas density- the rise in pressure due to the continuous gas in-flow and the fall in temperature due to heat loss to the cold tube wall. Mathematically, the rate of change of density is given by:

$$
\frac{\partial \rho}{\partial t}=\frac{1}{R T}\left(\frac{\partial p}{\partial t}-\frac{P}{T} \frac{\partial T}{\partial t}\right)
$$


The rise in pressure in the tube is known from the tube pressure vs. time measurement. Figure A3.1 shows $\partial P / \partial t \approx 8 \mathrm{kPa} / \mathrm{s}$ for the $100 \mathrm{kPa}$ run_LHe experiment. The rate of fall in the gas temperature, $\partial T / \partial t$ is not explicitly known but can be approximated in the following way. Assume the radial heat diffusion in the gas causes the temperature difference between the gas core (c) and the tube wall (w), $\Delta T_{c w}$ to evolve with time as $\Delta T_{c w}=\Delta T_{c w, 0} e^{-t / \tau_{c w}}$. Here $\Delta T_{c w, 0} \approx 290 \mathrm{~K}$ is the initial temperature difference and $\tau_{c w}=\left(\rho c_{P} / k\right) r^{2}$ is the time constant for radial heat diffusion $\left(\tau_{c w} \approx 0.8 s\right.$ for the tube pressure in figure A3.1). Writing the rate of gas cooldown as $\Delta T_{c w, 0} / \tau_{c w}$, the second term in equation A3.1 for the pressure data of figure A3.1 is $\approx 8 \mathrm{kPa} / \mathrm{s}$. The rate of change of density according to equation A3.1 is then $\approx 0.19 \mathrm{~kg} / \mathrm{m}^{3}$-s. Notice from figure A3.1 that the rate of pressure rise is nearly uniform over the tube length. Assuming $\Delta T_{c w, 0} / \tau_{c w}$ also remains nearly uniform along the tube, the rate of mass accumulating as gas averages to $\left(\pi D^{2} \Delta x / 4\right)(\Delta \rho / \Delta t)$. For the $100 \mathrm{kPa}$ run_LHe this rate is $2.2 \mathrm{E}-4 \mathrm{~kg} / \mathrm{s}$, which is about $1.2 \%$ of the inlet mass flow rate. Table A3.1 lists the inlet mass flow rates and the rates of gas mass accumulation for the three run_LHe experiments. Note that we have used T=295 K to evaluate equation A3.1, which has yielded conservative values of the gas mass accumulation rates. The actual gas temperature in the tube would be lower resulting into a higher average gas density and consequently will yield higher values of the gas mass accumulation rate.

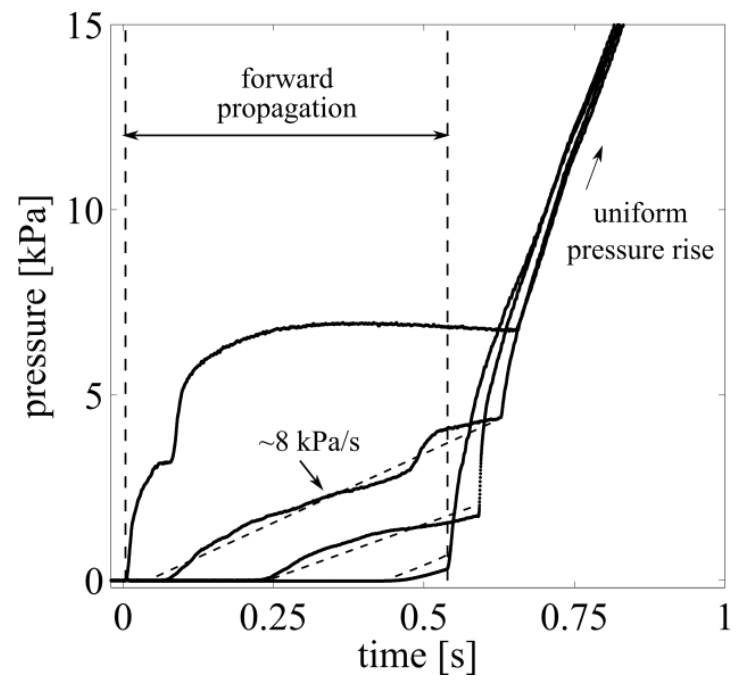

Figure A3.1: Pressure rise in the tube during the $100 \mathrm{kPa}$ run_LHe experiment. In the duration of propagation the pressure rise rate is about $8 \mathrm{kPa} / \mathrm{s}$ and is nearly uniform over the entire tube length.

Table A3.1 Mass in-flow rate compared to rate of rise of mass in gas phase

\begin{tabular}{cccc}
\hline $\begin{array}{c}\text { Starting pressure } \\
(\mathrm{kPa})\end{array}$ & $\begin{array}{c}\text { Mass in-flow rate } \\
\left(10^{-3} \mathrm{~kg} / \mathrm{s}\right)\end{array}$ & $\begin{array}{c}\text { Gas mass accumulation } \\
\left(10^{-3} \mathrm{~kg} / \mathrm{s}\right)\end{array}$ & $\%$ \\
\hline 50 & 8.1 & 0.13 & 1.6 \\
100 & 18.3 & 0.22 & 1.2 \\
150 & 27.7 & 0.34 & 1.2 \\
\hline
\end{tabular}




\section{References}

[1] T. Takiya, F. Higashino, Y. Terada, and A. Komura, "Pressure wave propagation by gas expansion in a high vacuum tube,” Journal of Vacuum Science and Technology- A, 17, 2059-2063 (1999).

http://dx.doi.org/10.1116/1.581726

[2] A. H. Shapiro, “The Dynamics and Thermodynamics of Compressible Fluid Flow”, vol. 2, $1^{\text {st }}$ ed., The Ronald Press (1954).

[3] T. Takiya, private communication.

[4] S. W. Van Sciver, “Helium Cryogenics”, $2^{\text {nd }}$ ed., Springer-Verlag, (2012).

[5] T. Boeckman et al., "Experimental Tests of Fault Conditions During the Cryogenic Operation of a XFEL Prototype Cryomodule,” Proceedings of the International Cryogenic Engineering Conference 22 International Cryogenic Materials Conference- 2008, Seoul (2009).

[6] A. A. Dalesandro, R. C. Dhuley, J. C. Theilacker, and S. W. Van Sciver, "Results from sudden loss of vacuum on scaled superconducting radio frequency cryomodule experiment," AIP Conference Proceedings, 1573, 1822 (2014). http://dx.doi.org/10.1063/1.4860929

[7] R. C. Dhuley and S. W. Van Sciver, “Sudden Vacuum Loss in Long Liquid Helium Cooled Tubes,” IEEE Transactions on Applied Superconductivity, Article\# 9000305, 25(3), (2015). http://dx.doi.org/10.1109/TASC.2014.2367156

[8] K. M. Welch, “Capture Pumping Technology: An Introduction,” $2^{\text {nd }}$ ed., Pergamon, (2011).

[9] R. C. Dhuley and S. W. Van Sciver, "Heat transfer in a liquid helium cooled vacuum tube following sudden vacuum loss,” IOP Conference Series: Material Science and Engineering, (to appear).

[10] G. Davey, “Cryopumping in the transitional and continuum pressure regions,” Vacuum, 26(1), 17-22 (1975). http://dx.doi.org/10.1016/S0042-207X(76)80414-9

[11] Data regression tool available at http://statpages.org/nonlin.html (last accessed July 13, 2015).

[12] J. E. A. John and T. G. Keith, “Gas Dynamics”, $3^{\text {rd }}$ ed., Prentice-Hall, (2006). 